\title{
Gravity, Parametric Resonance and Chaotic Inflation
}

\author{
Richard Easther ${ }^{1}$ and Matthew Parry ${ }^{2}$ \\ ${ }^{1}$ Department of Physics, Brown University, Providence, RI 02912, USA. \\ 2 Theoretical Physics Group, Blackett Laboratory, Imperial College, Prince Consort Rd, London, SW $2 B Z$, UK.
}

(October 19, 1999)

\begin{abstract}
We investigate the possibility that nonlinear gravitational effects influence the preheating era after inflation. Our work is based on numerical solutions of the inhomogeneous Einstein field equations, and is free of perturbative approximations. The one restriction we impose is to limit the inhomogeneity to a single spatial direction. We compare our results to perturbative calculations and to solutions of the nonlinear field equations in a rigid (unperturbed) spacetime, in order to isolate gravitational phenomena. We consider two types of initial conditions: where only one mode of the field perturbation has a non-zero initial amplitude, and where all the modes begin with a non-zero amplitude. Here we focus on preheating following inflation driven by a scalar field with a quartic potential. We confirm the broad picture of preheating obtained from the nonlinear field equations in a rigid background, but gravitational effects have a measurable impact on the dynamics for both sets of initial data. The rigid spacetime results predict that the amplitude of a single initially excited mode drops rapidly after resonance ends, whereas in the relativistic case the amplitude is roughly constant. With all modes initially excited, the longest modes in the simulation grow much more rapidly in the relativistic calculation than with a rigid background. However, we see no evidence for the sort of gravitational collapse associated with the formation of primordial black holes. The numerical codes described here are easily extended to more complicated resonant models, which we will examine in the future.
\end{abstract}

PACS numbers: 98.80.Cq 04.25.Dm BROWN-HET-1152 Imperial/TP/99-0/004

\section{INTRODUCTION}

For nearly a decade it has been known that coherent processes at the end of inflation can drive explosive particle production [1] via parametric resonance, leading to an era of preheating in the post-inflationary universe. Earlier assumptions about the transfer of energy from the inflaton to "regular" matter have been overturned, and the phenomenology of the post-inflationary universe is thus much richer than previously suspected. However, the corollary of such interesting behavior is understanding preheating has been a long and arduous task [2 15, 18, 16, 17, 19 28]. In particular, the problem is intrinsically nonlinear and takes place far from thermal equilibrium. Moreover, employing simplifying assumptions that are often useful in other circumstances can suppress crucial features of the preheating era, and when they are discarded the resulting predictions can change dramatically.

The simplest model to exhibit parametric resonance is chaotic inflation driven by the quartic potential,

$$
V(\phi)=\frac{\lambda}{4} \phi^{4}
$$

The $\phi$ particles themselves are produced parametrically, so there is no need to couple the inflaton to other bosons, which is a prerequisite for resonance when $V(\phi)=$ $m^{2} \phi^{2} / 2$. By shifting to conformal time and rescaling the field, all explicit dependence on the expanding background can be removed from the field's equation of motion. Consequently, the analysis of parametric resonance for $\lambda \phi^{4}$ is effectively performed in Minkowski space, and is thoroughly discussed by Greene et al. 111. The instability diagram contains a single, narrow resonance band and, to first order, co-moving modes which are initially in resonance appear to remain there for ever. Thus stochastic resonance, associated with a given wavelength passing through multiple resonance bands, does not occur. However, when the back reaction of the created particles on the field evolution is incorporated the resonant growth terminates [7, 11].

The inflaton field, $\phi$, is described by a homogeneous background part, $\phi_{0}(t)$, and an inhomogeneous part, $\delta \phi\left(t, x^{i}\right)$, which is usually expressed in terms of its Fourier modes, $\delta \phi_{k}$. Modes with wavenumbers, $k$, inside the resonance band grow exponentially, while other modes have oscillatory solutions. Because of the amplification of some of the $\delta \phi_{k}$, the "matter" develops a substantial inhomogeneous component. The background spacetime is usually assumed to be rigid, and therefore unperturbed.

A full treatment of parametric resonance must include the inhomogeneous metric induced by the inhomogeneous matter. Incorporating metric perturbations into the analysis of parametric resonance has been the subject of considerable recent attention [13, 14, 18, 22, 28]. Perturbation theory cannot give a full description of resonance, since once the $\delta \phi_{k}$ grow large, mode-mode couplings become significant. Consequently, a better understanding is obtained from a direct (numerical) solution of the nonlinear field equations. These equations are nonlinear due to the higher order terms in the potential, which are a necessary condition for resonance. However, in these simula- 
tions the background spacetime is an unperturbed FRW universe, so gravitational effects are implicitly ignored. To capture both the full nonlinear physics of preheating and any gravitational effects that influence the dynamics, we solve the evolution equations for the fields and the spacetime background. Our only a priori restriction is to confine the inhomogeneity to a single spatial dimension, which reduces the differential equations we must solve to a $1+1$ dimensional system.

Finally, it has been argued that second order terms in cosmological perturbation theory contribute to the overall dynamics via back reaction effects 29,30. In the context of preheating the one calculation which explicitly includes second order effects showed that they did not significantly affect the dynamics [26]. . The broader issue of the back reaction of perturbations on cosmological spacetimes can be addressed with our code, and we intend to pursue this topic in the future.

In general, we can anticipate two possible outcomes. If gravitational effects do not alter the dynamics the initial phase of the evolution of the metric perturbations will reproduce the results of gauge invariant perturbation theory 13,18,31,33, and at later times, when the perturbative limit is no longer valid, we will recover the results of the lattice simulations of the nonlinear field equations in a rigid background. On the other hand, if gravitational effects do play an important role, we will see phenomena that are not predicted by other approaches.

Two possible gravitational effects have been identified by previous work. On small scales, the increased inhomogeneity induced by resonance may lead to local gravitational collapse, and the formation of primordial black holes 22. Since they decay by emitting Hawking radiation, any such holes would provide an additional channel for thermalizing the post inflationary universe [34]. Many inflationary models do lead to the formation of primordial black holes, provided the perturbation spectrum they produce has more power at short scales than at COBE scales 35,36. However, if preheating creates a large amount of inhomogeneity at small scales, it could lead to primordial black hole formation in models where they would not otherwise be expected. On scales much larger than the post-inflationary Hubble length, it has been proposed that nonlinear effects enhance metric perturbations 25], potentially undermining the usual inflationary predictions for structure formation and the anisotropy of the microwave background. Consequently, during our analysis we are particularly interested in looking for evidence for both gravitational collapse at short length scales, and the enhancement of metric perturbations at long length scales. Finally, any changes in the initial resonance structure due to the inclusion of gravi-

\footnotetext{
${ }^{*}$ However, a very recent paper by Bassett et al. takes issue with these conclusions.
}

tational perturbations would be revealed by our simulations.

This paper extends the approach described by us in [24] (see also [37,38) in two ways. Firstly, we generalize the allowed range of initial data so that $\delta \phi$ can be an arbitrary function of position on the initial slice. Because of this, we can analyze the preheating era after chaotic inflation driven by a quartic potential, equation (1), with a realistic set of initial conditions. The further extension to models with more than one scalar field is straightforward and poses no new technical challenges. However, as the resonance provided by $\lambda \phi^{4}$ potential is confined to a narrow band, it provides an excellent laboratory for investigating both the perturbative and non-perturbative regimes, before we turn our attention to more complex two-field models, or single field models with more complex potentials.

In the following section, we assemble familiar results that describe the evolution of a homogeneous universe after the end of inflation. We then introduce the evolution equations for small perturbations, and the nonlinear equations of motion for the inhomogeneous fields in an unperturbed spacetime. In Section 3, we derive the specific form Einstein field equations for the inhomogeneous case, describe how we fix the initial conditions; we then outline the numerical algorithm we use to evolve the equations. Section 4 describes the results of our simulations. We look at two different sets of initial data. In the first, we start with a field perturbation that consists of single Fourier mode, chosen to lie inside the resonance band. This choice facilitates comparison with perturbation theory, by minimizing the effect of mode-mode couplings. The second, more realistic, choice of initial data is to begin with a field perturbation where all modes have a non-zero amplitude. Finally, in Section 5 we discuss our results and the questions they raise. For both the simulations, we find noticeable differences between the approximate calculations and the results obtained from solving the Einstein field equations. However, these do not appear to be large enough to overturn the overall picture obtained from lattice simulations of the nonlinear field equations in a static background.

\section{EVOLUTION EQUATIONS}

\section{A. Homogeneous Evolution}

For inflation in a flat, homogeneous universe, we have the general result

$$
\begin{aligned}
H^{2}=\left(\frac{\dot{a}}{a}\right)^{2} & =\frac{\kappa^{2}}{3}\left[\frac{\dot{\phi}^{2}}{2}+V(\phi)\right], \\
\dot{H} & =-\frac{\kappa^{2}}{2} \dot{\phi}^{2} \\
\ddot{\phi} & =-3 H \dot{\phi}-\frac{d V}{d \phi}
\end{aligned}
$$


where, as usual, $a(t)$ is the scale factor, and overdots stand for differentiation with respect to the time, $t$. The gravitational coupling is $\kappa^{2}=8 \pi G=8 \pi / m_{\mathrm{pl}}^{2}$. For the special case of a quartic potential, we make the transformation

$$
\eta=\int \frac{1}{a(t)} d t, \quad \chi=a \phi
$$

after which the constraint, equation (2), becomes

$$
a^{\prime 2}=\frac{\kappa^{2}}{3}\left[\frac{1}{2}\left(\chi^{\prime}-\chi \frac{a^{\prime}}{a}\right)^{2}+\frac{\lambda \chi^{4}}{4}\right],
$$

and $\chi$ obeys

$$
\chi^{\prime \prime}+\lambda \chi^{3}-\frac{a^{\prime \prime}}{a} \chi=0 .
$$

Primes refers to differentiation with respect to the conformal time, $\eta$. It is easy to show that the $\chi a^{\prime} / a$ and $\chi a^{\prime \prime} / a$ terms in equations (6) and (7) respectively quickly become negligible after the end of inflation [11].

\section{B. Linear Perturbation Theory}

The onset of resonance can be studied perturbatively. In many cases, the perturbative analysis of resonance is based on an expansion about the homogeneous solution for the field, and assumes that the spacetime background is unperturbed. However, including metric perturbations (which couple to perturbations in the matter) does not make the problem a great deal more difficult, and this is the approach we take here. Perturbation theory is most conveniently formulated using Mukhanov's variable, $Q$ [13, 18, 31, 32], which is related to (the $k$-th Fourier mode of) the gauge invariant perturbation $\Phi_{k}$ [31] by

$$
Q_{k}=\delta \phi_{k}^{(\mathrm{gi})}+\frac{\dot{\phi}}{H} \Phi_{k},
$$

where $\delta \phi_{k}^{(\mathrm{gi})}$ is the $k$-th Fourier mode of the gauge invariant perturbation of $\phi$, and $\Phi$ is the gauge invariant metric perturbation [31]. With $n$ fields we have $n$ distinct $Q$, which obey the coupled, second order equations 33

$$
\begin{aligned}
\ddot{Q}_{i}+ & 3 H \dot{Q}_{i}+\frac{k^{2}}{a^{2}} Q_{i}+\sum_{j=1}^{n}\left\{\frac{d^{2} V}{\partial \phi_{i} \partial \phi_{j}}\right. \\
-\kappa^{2} & {\left.\left[\left(3-\frac{\dot{H}}{H^{2}}\right) \dot{\phi}_{i} \dot{\phi}_{j}+\frac{1}{H} \frac{d}{d t}\left(\dot{\phi}_{i} \dot{\phi}_{j}\right)\right]\right\} Q_{j}=0 }
\end{aligned}
$$

where the subscript on $Q$ now labels the field, and not the Fourier mode.

The only relevant resonance band is 11

$$
k^{2}=\left.c^{2} \lambda a^{2} \phi^{2}\right|_{\dot{\phi}=0}
$$

where

$$
\frac{3}{2}<c^{2}<\sqrt{3}
$$

The time dependent quantities in equation (10) are all evaluated at an (arbitrary) moment when the kinetic energy of the inflaton vanishes. Strictly speaking, Greene et al. [11] do not analyze equation (9), since they assume that only the field is perturbed, and that there are no metric perturbations. However, the resonance structure is not significantly changed by the presence of metric perturbations.

Because of the scaling properties of the solution, the values of $k$ which fall inside the resonance band are not time dependent. This greatly simplifies the structure of resonance in this model, since only a small number of modes experience any resonant amplification. While we are interested the general question of nonlinear gravitational effects during preheating, this property of $\lambda \phi^{4}$ theory makes it an excellent testbed for exploring nonlinear gravitational effects during resonance, and the tools we are developing here will later be applied to more complicated models.

\section{Nonlinear Field Equations}

The next level of sophistication is to include all terms which are nonlinear in the fields, but to assume that the background spacetime is unperturbed. In the context of $\lambda \phi^{4}$ theory, this problem was first considered by Khlebnikov and Tkachev [7]. The perturbative analysis of preheating involves ordinary differential equations, but the nonlinear field equations are partial differential equations which are typically solved numerically on a spatial grid. Resonance causes individual modes to become large, so mode-mode couplings are significant and first order perturbation theory breaks down. The only nonlinear couplings ignored by these solutions are those attributable to gravity, and they thus provide a much more complete description of resonance and preheating than perturbation theory. The purpose of this paper is to find out whether this description is complete, and we do this by comparing solutions of the nonlinear field equations with the full relativistic calculation we describe in the next section.

We derive the nonlinear field equations by adding spatial gradient terms to equation (7),

$$
\chi^{\prime \prime}-\nabla^{2} \chi+\lambda \chi^{3}-\frac{a^{\prime \prime}}{a} \chi=0,
$$

where $\nabla=\partial_{i}$ and a prime denotes differentiation with respect to conformal time. Working with the conformal field, $\chi$, simplifies the equations. This equation includes gradients of $\chi$, but the metric is implicitly assumed to be a FRW spacetime. However, if the spatial gradients are non-zero, the metric cannot be perfectly homogeneous, 
so gravitational effects on preheating are ignored when this equation is solved.

Ignoring perturbations, the post-inflationary expansion mimics a radiation dominated universe, since on average the pressure and density of the oscillating field obey $p=\rho / 3$. In this limit, $a(\eta) \propto \eta$, and the $a^{\prime \prime} / a$ term rapidly becomes negligible after the oscillatory phase begins.

Our numerical code solves both the Einstein field equations and equation (12). All the nonlinear terms in equation (12) also appear in the relativistic equations. By comparing $\chi$ with the field evolution obtained from the relativistic calculation we can isolate gravitational effects during the preheating era.

To recover $\phi$ from our solution for equation (12) we divide $\chi$ by $a$. In order to obtain $a$, one could solve the ordinary differential equations which give $a$, or assume an exact radiation dominated expansion. In practice we extracted $a$ by averaging the metric functions derived from our numerical solutions of the full Einstein equations. Since the average of the perturbation vanishes, we recover the background solution in a self-consistent manner. However, we followed [7] and dropped the $a^{\prime \prime} / a$ term from equation (12).

\section{EINSTEIN FIELD EQUATIONS}

We now turn our attention to the explicit form of the Einstein field equations which we will solve numerically. To reduce the computational complexity of the problem, we assume that the universe has a planar symmetry; the metric functions depend only on $t$ and $z$, and are independent of $x$ and $y$. In 24, we examined possible nonlinear gravitational effects after $m^{2} \phi^{2}$ inflation using the metric

$$
d s^{2}=d t^{2}-A^{2}(t, z) d z^{2}-B^{2}(t, z)\left(d x^{2}+d y^{2}\right),
$$

which describes an inhomogeneous universe in which the $d x^{2}+d y^{2}$ sections have zero spatial curvature. In principle, we could retain this metric here, but in practice it is simpler to work with a metric for which the field equations reduce to the conformal time version of the homogeneous system, rather than physical time which is the homogeneous limit of equation (13). The oscillation time for the homogeneous field is constant when expressed in conformal time, and this property persists in the nonlinear system. Thus the time-scale governing the evolution does not change significantly during the simulation, and we can use a constant timestep, simplifying our numerical problem.

Specifically, a co-ordinate transformation from $t$ and $z$ to $\eta$ and $\zeta$ allows us to re-write equation (13) as:

$$
d s^{2}=\alpha^{2}(\eta, \zeta)\left(d \eta^{2}-d \zeta^{2}\right)-\beta^{2}(\eta, \zeta)\left(d x^{2}+d y^{2}\right) .
$$

With this metric, the non-trivial components of the Einstein tensor are

$$
\begin{aligned}
G_{\eta}^{\eta} & =\frac{1}{\alpha^{2}}\left[2 \frac{\beta, \zeta \zeta}{\beta}-\frac{\beta_{, \eta}^{2}}{\beta^{2}}+\frac{\beta_{, \zeta}^{2}}{\beta^{2}}-2 \frac{\alpha_{, \eta} \beta_{, \eta}}{\alpha \beta}-2 \frac{\alpha_{, \zeta} \beta_{, \zeta}}{\alpha \beta}\right], \\
G_{\zeta}^{\eta} & =\frac{1}{\alpha^{2}}\left[2 \frac{\beta, \eta \zeta}{\beta}-2 \frac{\alpha_{, \eta} \beta_{, \zeta}}{\alpha \beta}-2 \frac{\alpha_{, \zeta} \beta, \eta}{\alpha \beta}\right], \\
G_{\zeta}^{\zeta} & =\frac{1}{\alpha^{2}}\left[-2 \frac{\beta_{, \eta \eta}}{\beta}-\frac{\beta_{, \eta}^{2}}{\beta^{2}}+\frac{\beta_{, \zeta}^{2}}{B^{2}}+2 \frac{\alpha_{, \eta} \beta_{, \eta}}{\alpha \beta}+2 \frac{\alpha_{, \zeta} \beta, \zeta}{\alpha \beta}\right], \\
G_{x}^{x} & =\frac{1}{\alpha^{2}}\left[-\frac{\alpha, \eta \eta}{\alpha}-\frac{\beta_{, \eta \eta}}{\beta}+\frac{\alpha_{, \zeta \zeta}}{\alpha}+\frac{\beta_{, \zeta \zeta}}{\beta}+\frac{\alpha_{, \eta}^{2}}{\alpha^{2}}-\frac{\alpha_{, \zeta}^{2}}{\alpha^{2}}\right], \\
G_{y}^{y} & =G_{x}^{x} .
\end{aligned}
$$

The field Lagrangian is,

$$
\mathcal{L}=\frac{1}{2} \frac{\phi_{, \eta}^{2}-\phi_{, \zeta}^{2}}{\alpha^{2}}-V(\phi),
$$

and yields the following equation of motion for $\phi$

$$
\phi_{, \eta \eta}=-2 \frac{\beta_{, \eta}}{\beta} \phi_{, \eta}+\phi_{, \zeta \zeta}+2 \frac{\beta, \zeta}{\beta} \phi_{, \zeta}-V_{, \phi} \alpha^{2} .
$$

The components of the energy-stress-momentum tensor are therefore

$$
\begin{aligned}
T_{\eta}^{\eta} & =\frac{1}{2 \alpha^{2}}\left(\phi_{, \eta}^{2}+\phi_{, \zeta}^{2}\right)+V, \\
T_{\zeta}^{\eta} & =\frac{1}{\alpha^{2}} \phi_{, \eta} \phi_{, \zeta}, \\
T_{\zeta}^{\zeta} & =-\frac{1}{2 \alpha^{2}}\left(\phi_{, \eta}^{2}+\phi_{, \zeta}^{2}\right)+V, \\
T_{x}^{x}=T_{y}^{y} & =-\frac{1}{2 \alpha^{2}}\left(\phi_{, \eta}^{2}-\phi_{, \zeta}^{2}\right)+V .
\end{aligned}
$$

The corresponding field equations are

$$
\begin{aligned}
\alpha_{, \eta \eta}= & -\frac{\alpha_{, \eta} \beta_{, \eta}}{\beta}+\frac{\alpha \beta_{, \eta}^{2}}{2 \beta^{2}}+\frac{\alpha \beta,_{\zeta \zeta}}{\beta}-\frac{\alpha \beta_{, \zeta}^{2}}{2 \beta^{2}}-\frac{\alpha_{, \zeta} \beta, \zeta}{\beta}+ \\
& \alpha_{, \zeta \zeta}+\frac{\alpha_{, \eta}^{2}}{\alpha}-\frac{\kappa^{2} \alpha}{2}\left[\frac{1}{2} \phi_{, \eta}^{2}-\frac{3}{2} \phi_{, \zeta}^{2}-V \alpha^{2}\right] \\
\beta_{, \eta \eta}= & -\frac{\beta_{, \eta}^{2}}{2 \beta}+\frac{\beta_{, \zeta}^{2}}{2 \beta}+\frac{\alpha_{, \eta} \beta_{, \eta}}{\alpha}+\frac{\alpha_{, \zeta} \beta}{\alpha}- \\
& \frac{\kappa^{2} \beta}{2}\left[\frac{1}{2} \phi_{, \eta}^{2}+\frac{1}{2} \phi_{, \zeta}^{2}-V \alpha^{2}\right]
\end{aligned}
$$

Finally, we have two constraints which must impose on the initial data, but which are then satisfied at all later times as a consequence of the field equations,

$$
\begin{aligned}
& \frac{\beta_{, \eta \zeta}}{\beta}-\frac{\alpha_{, \eta} \beta_{, \zeta}}{\alpha \beta}-\frac{\alpha_{, \zeta} \beta, \eta}{\alpha \beta}=-\frac{\kappa^{2}}{2} \phi_{, \eta} \phi_{, \zeta} . \\
- & \frac{\beta_{, \eta}^{2}}{2 \beta^{2}}-\frac{\alpha_{, \eta} \beta, \eta}{\alpha \beta}+\frac{\beta, \zeta \zeta}{\beta}+\frac{\beta_{, \zeta}^{2}}{2 \beta^{2}}-\frac{\alpha_{, \zeta} \beta_{, \zeta}}{\alpha \beta} \\
= & -\frac{\kappa^{2}}{2}\left[\frac{1}{2} \phi_{, \eta}^{2}+\frac{1}{2} \phi_{, \zeta}^{2}+V \alpha^{2}\right] .
\end{aligned}
$$




\section{A. Initial Conditions}

To solve the equations, we must specify the initial conditions. These cannot all be chosen arbitrarily, since the initial data must satisfy the constraint equations. We consider two possible scenarios. In the first we excite a single mode and track its evolution using the full nonlinear equations. In the second, all modes are initially excited. Using the first approach it is easy to compare our results to a perturbative calculation. However, beginning with a configuration in which all the inhomogeneous modes have a small, non-zero amplitude is more realistic.

\section{A Single Excited Mode}

If we only wish to excite a single mode, we can pick initial conditions which ensure that the constraints are automatically satisfied, and thus do not need to be solved explicitly before beginning the actual integration. Following the approach of 24], we set $\alpha(0, \zeta)=\beta(0, \zeta)=1$, $\phi(0, \zeta)=\phi_{0}$. Equation (21) is solved if

$$
\begin{aligned}
& \alpha_{, \eta}(0, \zeta)=\frac{\kappa^{2}}{2 C}\left(\frac{\phi_{, \eta}^{2}}{2}+V\left(\phi_{0}\right)\right)-\frac{C}{2}, \\
& \beta_{, \eta}(0, \zeta)=\sqrt{\frac{\kappa^{2}}{3}\left(\frac{\left\langle\phi_{, \eta}^{2}\right\rangle}{2}+V\left(\phi_{0}\right)\right)}=C .
\end{aligned}
$$

Our choice of $C$ ensures $\left\langle\alpha_{, \eta}(0, \zeta)\right\rangle=\left\langle\beta_{, \eta}(0, \zeta)\right\rangle$, where $\langle\cdots\rangle$ is a spatial average. The actual inhomogeneity is injected through the inflaton velocity, which we choose to be

$$
\phi_{, \eta}(0, \zeta)=\Pi+\epsilon \sin \left(\frac{2 \pi k \zeta}{Z}\right),
$$

where $Z$ is the length of our "box", and $\Pi$ is the average initial velocity. However, since we have assumed that $\phi$, $\alpha$ and $\beta$ are independent of $\zeta$ on our initial slice we can only excite multiple modes if we assume that they are all initially in phase with each other.

\section{General Initial Data}

In the second scenario, where all the inhomogeneous modes are initially excited, we want to allow their initial phases to be random. With just one mode the phase is irrelevant, but demanding that all modes begin with correlated phases would lead to an unacceptable loss of generality. In particular, we are interested in modeling the Gaussian fluctuations predicted by inflation, which are defined in part by having random phases. In this case there is no simple and obvious approach to solving the constraints. A general procedure does exist to fix the initial data in $3+1$ dimensional numerical relativity
[39], but it is not necessarily easy to implement. Since we are interested in a perturbation that is initially small, we will adopt the approach used by Goldwith and Piran [40], who simulate the onset of inflation in a $1+1$ dimensional universe. Their approach is to add a small amount of radiation, with a spatial dependence chosen to ensure that the overall density perturbation vanishes on the initial surface. Having done this the constraints become simple to solve, while the radiation introduced by this procedure always makes a trivial contribution to the overall density, and quickly decays away.

We modify Goldwirth and Piran's approach slightly, and use an uncoupled scalar field, $\psi$, instead of radiation. The energy density of a massless free field drops as $a^{-6}$ in a homogeneous universe, in contrast to radiation which scales as $a^{-4}$. This ensures that the contribution from $\psi$ to the overall density drops more quickly than that of $\phi$, which behaves like radiation.

Including a second field in our code is simple. The field itself obeys the equation of motion,

$$
\psi_{, \eta \eta}=-2 \frac{\beta_{, \eta}}{\beta} \psi_{, \eta}+\psi_{, \zeta \zeta}+2 \frac{\beta_{, \zeta}}{\beta} \psi_{, \zeta},
$$

and we modify equations (18) and (19) by adding $\psi_{, \zeta}$ and $\psi_{, \eta}$ terms analogous to the derivatives of $\phi$ that are already there. Most importantly for us, the revised constraints are

$$
\begin{aligned}
& \frac{\beta_{, \eta \zeta}}{\beta}-\frac{\alpha_{, \eta} \beta_{, \zeta}}{\alpha \beta}-\frac{\alpha_{, \zeta} \beta_{, \eta}}{\alpha \beta} \\
= & -\frac{\kappa^{2}}{2}\left(\phi_{, \eta} \phi_{, \zeta}+\psi_{, \eta} \psi_{, \zeta}\right), \\
& -\frac{\beta_{, \eta}^{2}}{2 \beta^{2}}-\frac{\alpha_{, \eta} \beta_{, \eta}}{\alpha \beta}+\frac{\beta_{, \zeta \zeta}}{\beta}+\frac{\beta_{, \zeta}^{2}}{2 \beta^{2}}-\frac{\alpha_{, \zeta} \beta_{, \zeta}}{\alpha \beta} \\
= & -\frac{\kappa^{2}}{2}\left[\frac{1}{2} \phi_{, \eta}^{2}+\frac{1}{2} \phi_{, \zeta}^{2}+\frac{1}{2} \psi_{, \eta}^{2}+\frac{1}{2} \psi_{, \zeta}^{2}+V \alpha^{2}\right] .
\end{aligned}
$$

We solve these equations on the initial slice by choosing $\psi_{, \zeta}$ and $\psi_{, \eta}$ so that the right hand sides of the constraints simplify, or

$$
\begin{aligned}
\phi_{, \eta} \phi_{, \zeta}+\psi_{, \eta} \psi_{, \zeta} & =0, \\
\frac{1}{2} \phi_{, \eta}^{2}+\frac{1}{2} \phi_{, \zeta}^{2}+\frac{1}{2} \psi_{, \eta}^{2}+\frac{1}{2} \psi_{, \zeta}^{2}+V \alpha^{2} & =D^{2} .
\end{aligned}
$$

Now we are free to choose

$\alpha(0, \zeta)=\beta(0, \zeta)=1, \quad \alpha_{, \eta}(0, \zeta)=\beta_{, \eta}(0, \zeta)=H$

where $H=D \kappa / \sqrt{3}$. Adding equation (27) to equation (29) gives two coupled equations for $\psi_{, \eta}$ and $\psi_{, \zeta}$, which we solve to find

$$
\begin{aligned}
& \psi_{, \eta}=\frac{A \pm \sqrt{A^{2}-4 B}}{2}, \\
& \psi_{, \zeta}=\frac{A \mp \sqrt{A^{2}-4 B}}{2},
\end{aligned}
$$


where $A^{2}=2 D^{2}-2 V-\left(\phi_{, \eta}+\phi_{, \zeta}\right)^{2}$ and $B=-\phi_{, \zeta} \phi_{, \eta}$, and we have used $\alpha(0, \zeta)=1$. We can choose either of the two solutions. Finally, for these to be real, we need $A^{2}-4 B>0$, which is equivalent to demanding

$$
D^{2} \geq \max \left[\frac{1}{2}\left(\left|\phi_{, \eta}\right|+\left|\phi_{, \zeta}\right|\right)^{2}+V\right] .
$$

We are free to choose $D^{2}$, subject to the above constraint. In practice we want to minimize the impact of the extra field, so we set it close to the lower bound. However, our numerical scheme is somewhat more stable if the inequality is not completely saturated, and in practice we chose a value of $D$ somewhat larger than the minimum allowable value. Finally, $\psi_{, \zeta}$ and $\psi_{, \eta}$ are periodic, as required by the boundary conditions.

\section{Choice of Initial Data}

We need to specify $\phi$ and $\phi_{, \eta}$ on the initial slice. We begin our simulations at the end of inflation, so $\phi$ is described by small fluctuations overlaid on top of the homogeneous zero mode. The fluctuations are quantum in origin, and are generated by the usual inflationary mechanism. In practice the spectrum will have some scale dependence, especially towards the end of inflation when the slow roll approximation breaks down. If we wished we could track the perturbations from their inception during inflation, but we will assume the spectrum is initially scale-free since our results do not depend on the precise form of the spectrum.

Let $q=\langle\phi(0, \zeta)\rangle$ and $p=\left\langle\phi_{, \eta}(0, \zeta)\right\rangle$, where $\langle\cdots\rangle$ denotes a spatial average; these values are chosen to be those at the end of inflation. We will write the perturbation in $\phi$, for any time, as

$$
X=\beta(\phi-\langle\phi\rangle) \text {. }
$$

Since, the metric perturbations which are first order in $\eta$ vanish when $\eta=0$, we can expand equation (17) to obtain an equation for the homogeneous mode and a linear equation for $X$, valid on the initial slice when $\eta=0$. Taking the Fourier transform of this latter equation gives

$$
X_{\mathbf{k}, \eta \eta}+\left(k^{2}+H^{2}+\left\langle V_{, \phi \phi}-\kappa^{2} V\right\rangle\right) X_{\mathbf{k}}=0 .
$$

Thus for a short time near $\eta=0$ each $k$-mode of $X$ can be approximated as a simple harmonic oscillator with a $k$-dependent frequency,

$$
\omega_{k}^{2}=k^{2}+H^{2}+\left\langle V_{, \phi \phi}-\kappa^{2} V\right\rangle
$$

Our approach will be to treat $X(0, \zeta)$ as a free quantum field in the vacuum state; this amounts to the initial choice

$$
\begin{aligned}
X_{\mathbf{k}} & =\frac{1}{\sqrt{2 \omega_{k}}}|n| e^{2 \pi i r}, \\
X_{\mathbf{k}, \eta} & =-i \omega_{k} X_{\mathbf{k}},
\end{aligned}
$$

where $n$ is a number taken randomly from a Gaussian distribution of mean 0 and variance 1 , and $r$ is taken randomly from the interval $[0,1]$. For equations (37) and (38) to be applicable the adiabatic limit must hold, namely $\omega_{k, \eta} \ll \omega_{k}^{2}$. A more rigorous treatment is possible but our approach is adequate for fixing the scale of the fluctuations. Moreover, we emphasize that while this choice of initial conditions is a good approximation when applied prior to the onset of resonance, its underlying assumptions will be strongly violated at later times.

We insert $\phi$ into equation (36) to determine $\omega_{k}$, from which we learn $X_{\mathbf{k}}$ and, ultimately, $\phi$. If we insist the fluctuations are smallif we can solve this problem iteratively. We approximate $\left\langle V_{, \phi \phi}-\kappa^{2} V\right\rangle$ by $V_{, \phi \phi}(q)-\kappa^{2} V(q)$. The final complication is that $H$ also depends on $\phi$ through equation (29) and equation (30). Our approach here is as follows: $H^{2}=D^{2} \kappa^{2} / 3$, and $D^{2}=\rho_{\phi}+\rho_{\psi}$ is a constant, so $D^{2}=\left\langle\rho_{\phi}+\rho_{\psi}\right\rangle$. We approximate $\left\langle\rho_{\phi}\right\rangle$ by $p^{2} / 2+V(q)$ and then choose an $\epsilon$ and insist $\left\langle\rho_{\psi}\right\rangle=\epsilon\left\langle\rho_{\phi}\right\rangle$. Setting $\epsilon \ll 1$ ensures the initial energy density of $\psi$ is small and fixes $D$ and $H$. However, if $\epsilon$ is too small it will not satisfy equation (33). This can only be checked when $\phi$ and $\phi_{, \eta}$ have been determined: if $\epsilon$ is too small and one must repeat the process with a larger value. In practice $\epsilon$ can be on the order of $\frac{1}{10}$, and the contribution of $\psi$ rapidly becomes negligible as the universe expands.

Once $X(0, \zeta)$ and $X_{, \eta}(0, \zeta)$ have been found though, it is a simple matter to obtain the initial conditions for $\phi$ and $\phi, \eta$ using equation 34 :

$$
\begin{aligned}
\phi(0, \zeta) & =q+X(0, \zeta), \\
\phi_{, \eta}(0, \zeta) & =p+X_{, \eta}(0, \zeta)-H X(0, \zeta) .
\end{aligned}
$$

\section{B. Coordinate Transformation}

To transform between the "conformal" and "physical" co-ordinates, we need $t=t(\eta, \zeta)$ and $z=z(\eta, \zeta)$. The transformation that connects equation (14) to equation (13) reduces to the following pair of differential equations

$$
\begin{aligned}
t_{, \eta} & =\sqrt{t_{, \zeta}^{2}+\alpha^{2}(\eta, \zeta)}, \\
z_{, \eta} & =\frac{t_{, \zeta} z_{, \zeta}}{t_{, \eta}}
\end{aligned}
$$

from which we find

\footnotetext{
${ }^{\dagger}$ In a numerical scheme, requiring small fluctuations leads to a constraint on the lattice spacing. This is because the lattice provides a natural UV (and IR) cutoff to the unrenormalized quantum field theory we have effectively introduced in equation (37) and equation (38).
} 


$$
\begin{aligned}
& A(t, z)=A(t(\eta, \zeta), z(\eta, \zeta))=\frac{t_{, \eta}}{z, \zeta}, \\
& B(t, z)=B(t(\eta, \zeta), z(\eta, \zeta))=\beta(\eta, \zeta),
\end{aligned}
$$

and the specification is completed with the following set of initial conditions,

$$
t(0, \zeta)=0, \quad z(0, \zeta)=\zeta .
$$

Since both $t$ and $z$ are transformed, slices of constant $\eta$ in the conformal frame are not mapped directly to slices of constant $t$ in the physical frame.

As we have already noted, the $(\eta, \zeta)$ co-ordinates are convenient because the typical timescale for the field oscillations is roughly constant in this frame. Moreover, the degree of spatial variation is $\alpha(\eta, \zeta)$ is significantly less than in the corresponding $A(t, z)$, which is enhances our numerical stability. In practice, we solve equations (43) and (44) alongside with the field equations themselves, thereby obtaining solutions for both forms of the metric.

\section{Numerical Code}

The actual numerical code is essentially the same as that used in 24. The simulations are evolved forwards in time on an $N$ point spatial grid, with periodic boundary conditions. We use a fourth-order differencing scheme to express equations (18) and (19) as $N$ ordinary differential equations, and solve these using a fourth-order RungeKutta integrator. Numerical noise is controlled with a dealiasing algorithm, which requires filtering Fourier components with wavelengths less than 8 grid spacings at each timestep, so our actual spatial resolution is effectively 16 times larger than the separation of the individual points, $\delta \zeta[41,42$. We track the numerical accuracy of the solutions by studying how closely the constraint equations are satisfied, and have also compared solutions obtained directly from the metric equation (13), and those obtained with equation (14) and the co-ordinate transformation equations (43) and (44).

The Fourier transforms needed for de-aliasing are carried out using the Fast Fourier Transform algorithm. While this works for any value of $N$, it is most efficient when $N$ is an integer power of 2 .

We choose our units so that $\kappa^{2}=\lambda=1$. Setting $\kappa^{2}$ defines the energy scale, but on first sight choosing $\lambda=1$ may seem to result in a loss of generality. However, all terms in the equations of motion which do not involve $V$ or $V_{, \phi}$ contain two derivatives with respect to $\eta$ and/or $\zeta$ and the rescaling $\eta \rightarrow \eta / \sqrt{\lambda}, \zeta \rightarrow \zeta / \sqrt{\lambda}$ ensures that all the terms in the equations of motion are proportional to $\lambda$. Consequently, our choice of $\lambda$ is equivalent to a rescaling of time and length. All the values of $\zeta$ and $\eta$ given in our results reflect this scaling. Finally, when we plot the spectra for variables such as $\Phi$ and $Q$ we normalize $k$ so that $k=1$ corresponds to the mode whose wavelength is equal to the initial Hubble radius.

\section{The Perturbative Limit}

Consider the connection between equation (14) and the familiar gauge invariant theory of cosmological perturbations [31. In conformal time, the most general metric that describes scalar perturbations to a FRW metric with flat spatial sections and whose spatial dependence is restricted to the $\zeta$ direction is

$$
\begin{aligned}
d s^{2}= & a(\eta)^{2}\left\{(1+2 \varphi(\eta, \zeta)) d \eta^{2}-2 b(\eta, \zeta)_{, i} d x^{i} d \eta\right. \\
& \left.-\left[(1-2 \psi(\eta, \zeta)) \delta_{i j}+2 E(\eta, \zeta)_{, i j}\right]\right\} d x^{i} d x^{j} .
\end{aligned}
$$

By comparing this with equation (14) and using the definition of the gauge invariant perturbation, $\Phi$, 31,24] we obtain

$$
\Phi_{, \zeta \zeta}=\varphi_{, \zeta \zeta}-\frac{1}{a} \frac{d}{d \eta}\left(E_{, \eta \zeta \zeta} a\right)
$$

or

$$
\Phi_{, \zeta \zeta}=\varphi_{, \zeta \zeta}-\frac{1}{a} \frac{d}{d \eta}\left[\frac{\alpha_{, \eta} \alpha-\beta_{, \eta} \beta}{a}-H\left(\alpha^{2}-\beta^{2}\right)\right] .
$$

In order to obtain $Q$, we also need the gauge invariant perturbation, $\delta \phi^{(\mathrm{gi})}$. For our metric [31],

$$
\delta \phi^{(\mathrm{gi})}=\delta \phi-\frac{d \phi_{0}}{d \eta} E_{, \eta},
$$

where $\delta \phi$ is the gauge dependent perturbation measured at constant $\eta$. After differentiating twice with respect to $\zeta$, and again comparing equation (46) with equation (14) we find that

$$
\delta \phi_{, \zeta \zeta}^{(\mathrm{gi})}=\delta \phi_{, \zeta \zeta}-\frac{1}{a^{2}} \frac{d \phi_{0}}{d \eta}\left(\alpha \alpha_{, \eta}-\beta \beta_{, \eta}-\frac{a_{, \eta}}{a}\left(\alpha^{2}-\beta^{2}\right)\right) .
$$

Our numerical simulations thus give the second derivatives of $\Phi$ and $\delta \phi^{(\mathrm{gi})}$ with respect to $\zeta$. To compare our results with perturbation theory, we need the Fourier transforms of $\Phi$ and $\delta \phi^{(\mathrm{gi})}$. We find these by transforming $\Phi_{, \zeta \zeta}$ and $\delta \phi_{, \zeta \zeta}^{(\mathrm{gi})}$, and multiplying by $-k^{2}$ which, thanks to the general properties of the Fourier transform, yields $\Phi_{k}$ and $\delta \phi_{k}^{(\mathrm{gi})}$. We obtain the background variables $\left(a(\eta), \phi_{0}(\eta)\right)$ by averaging over the spatial grid at each value of $\eta$. Once this is done, we have all the raw materials we need to calculate $Q_{k}$ from equation (8), and make the comparison with perturbation theory. In models with more than one field, each field has a gauge invariant perturbation analogous to $\delta \phi^{(\mathrm{gi})}$.

\section{RESULTS}

\section{A. A Single Excited Mode}

We begin our analysis with an initial field field perturbation that consists of a single mode, chosen to lie within 


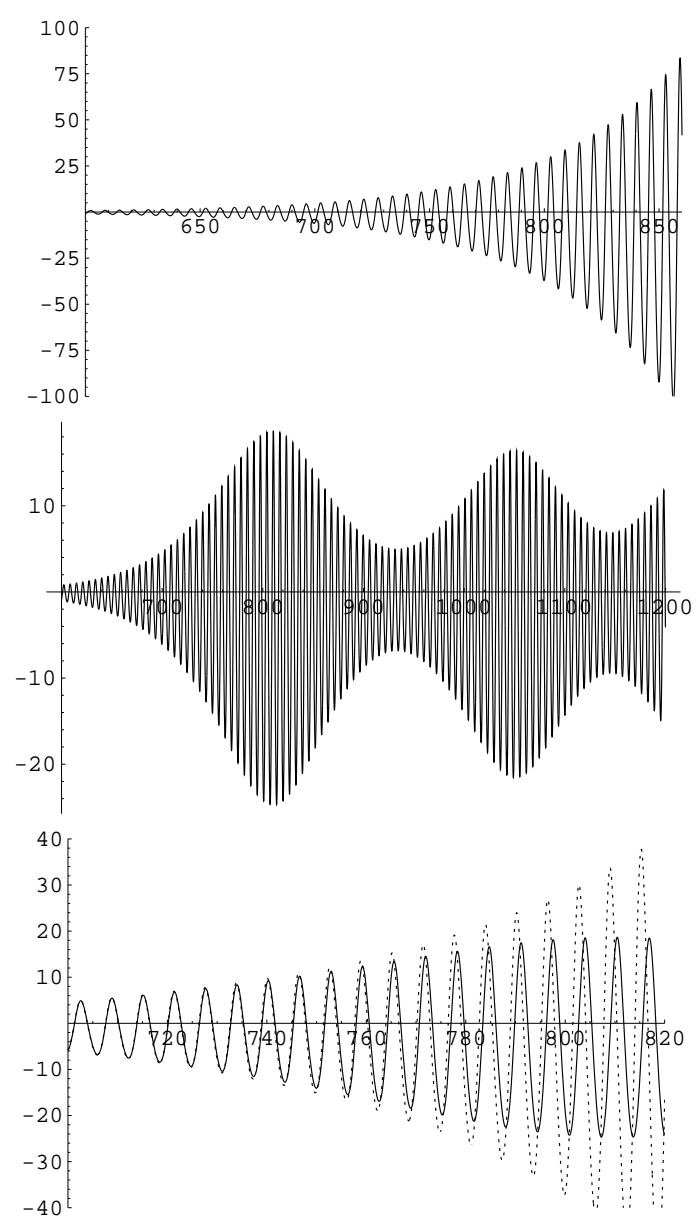

FIG. 1. The evolution of a single resonant mode is plotted as a function of $\eta$. The top panel shows the evolution of $Q_{\phi}$ predicted by perturbation theory. The second plot shows the evolution of $Q_{\phi}$ derived from the full Einstein field equations. The third panel superimposes the perturbative and non-perturbative results at the moment when perturbation theory breaks down.

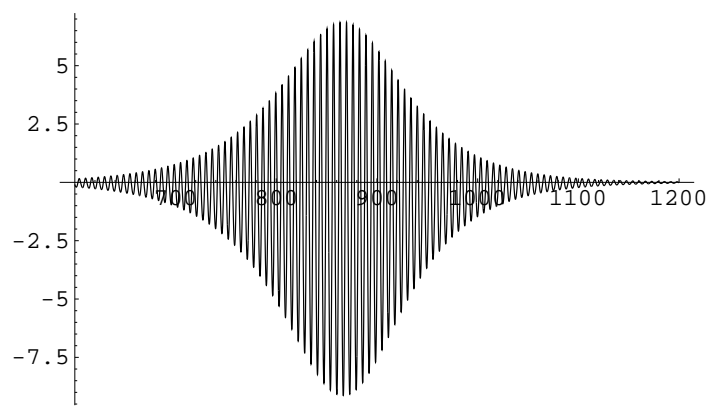

FIG. 2. We plot the evolution of $Q_{\phi}(\chi / a)$ derived from the nonlinear field equations with a static background. The specific mode plotted is the same as that shown in fig. (1), but the normalization is different since we dropped the $a^{\prime \prime} / a$ term before solving equation (12). the resonance band. The parameters for the integration are

$$
\begin{gathered}
N=65336=2^{16}, \delta \eta=\delta \zeta=0.0722, k_{r e s}=583, \\
\phi_{0}=-0.58132, \dot{\phi}_{0}=0
\end{gathered}
$$

where $k_{\text {res }}$ denotes the mode number of the single excited mode, and $k=1$ corresponds to the mode whose wavelength is equal in size to the box. The values for $\phi_{0}$ and $\dot{\phi}_{0}$ correspond to the first turn-around point after inflation. In the plots we normalize $k$ so that $k=1$ corresponds to a mode with a wavelength equal to the initial Hubble horizon size, and $k_{r e s} \sim 1.2$. The initial field values correspond to the field being at the end of the first oscillation it makes after the end of inflation. With this value of $N$, approximately 4000 distinct Fourier modes can be resolved after de-aliasing. The spacing between the points, $\delta \zeta$, ensures that the simulation volume initially contains approximately 500 distinct Hubble volumes. As the comoving wavelength of the resonant modes is slightly less than the Hubble length at the end of the inflation we can resolve modes with frequencies up to 7 times higher than the frequencies of modes in the resonance band.

Fig. (1) contrasts the perturbative evolution of our single excited mode, found by solving equation (9), with the $Q_{\phi}$ extracted from the relativistic calculation. Initially, perturbation theory is valid and $Q$ increases exponentially. While perturbation theory predicts that exponential growth continues indefinitely, non-perturbative effects eventually terminate the resonance, as shown in the plots. The termination of resonance is not attributable to gravitational effects, and is well known from studies of equation (12).Fig. (2) shows $Q_{\phi}$ obtained by solving equation (12), which assumes that $\Phi=0$ and so $Q$ reduces to $\delta \phi$. While the resonance terminates, the subsequent evolution differs from the relativistic result, since in the absence of gravity the amplitude of the perturbation decreases after the end of resonance. Conversely, the $Q_{\phi}$ obtained from the full relativistic calculation remains comparatively large after the resonant growth terminates. Presumably this is due to the self-gravity of the perturbation, which only enters perturbation theory at second order.

We do not expect the solutions for $\chi / a$ and $\phi$ to overlap exactly since we have dropped the $a^{\prime \prime} / a$ term from equation (12). This term quickly becomes irrelevant, but its absence during the initial stages of the evolution means that a perfect match is not possible, even with the same initial conditions. Moreover, this calculation includes the "compensating" field $\psi$, even though it is not strictly necessary with a single mode and this also changes the initial normalization of $\phi$, relative to $\chi / a$.

In addition to terminating the resonance, nonlinear effects induce mode-mode couplings which transfer power to modes outside the resonance band. This can be seen in fig. (3), where we plot the power spectrum of the gauge invariant perturbation, $\Phi$. As we specify the initial perturbation in a specific gauge, the corresponding gauge 

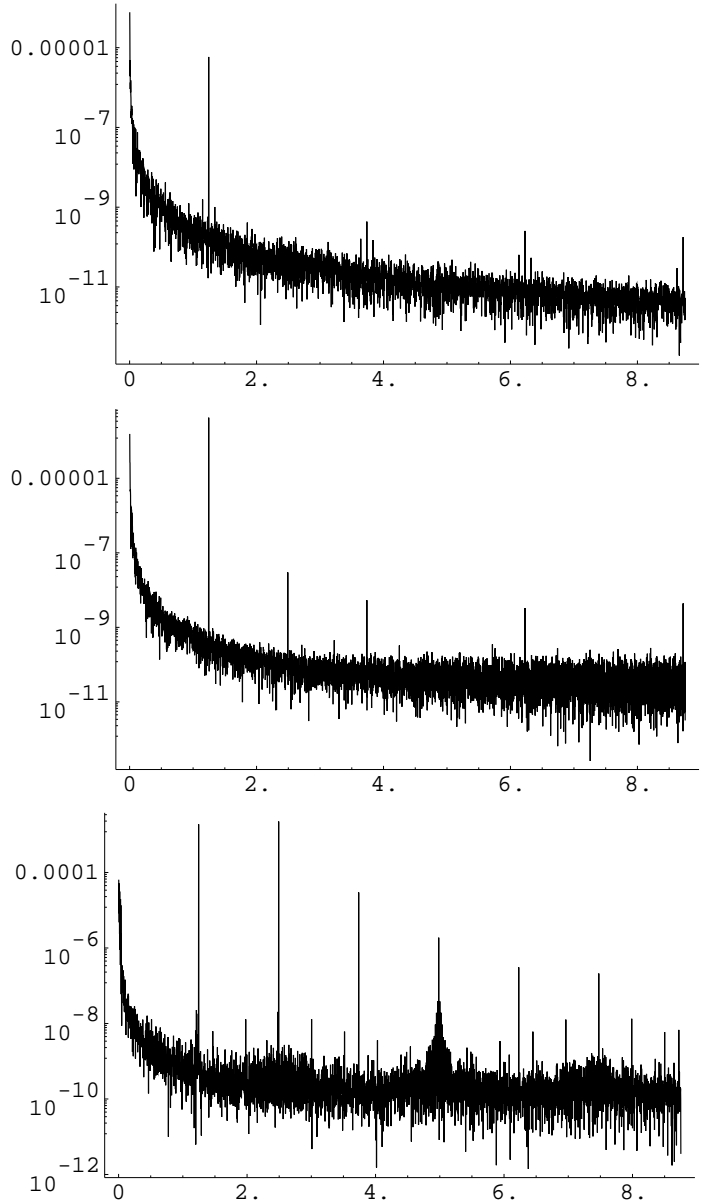

FIG. 3. The power spectrum, $\left|\Phi_{k}\right|$ of the gauge-invariant metric perturbation is plotted against $k$. A single resonant mode is initially excited, but since we specify the initial perturbation in a specific gauge, all modes of $\Phi$ have some initial power. The plots show $\left|\Phi_{k}\right|$ at $\eta=0.22,414.8$ and 902.8 . The $x$-axis is scaled to that a mode with a wavelength equal to the initial Hubble radius has $k=1$.

invariant quantity actually possesses some power in all modes. As time progresses, the nonlinear couplings amplify the "harmonics" of the resonant mode. This effect is also apparent when we solve equation (12), but the quantitative results differ from the general relativistic calculation due to the observed decay of the perturbations in the absence of gravity.

\section{B. General Initial Conditions}

Having explored the nonlinear evolution found when a single mode of the perturbation was initially excited, we turn to the more realistic case in which the initial amplitude of all modes is non-zero. The initial amplitudes are set using equations (37) and (38), while the parameters for the simulation were
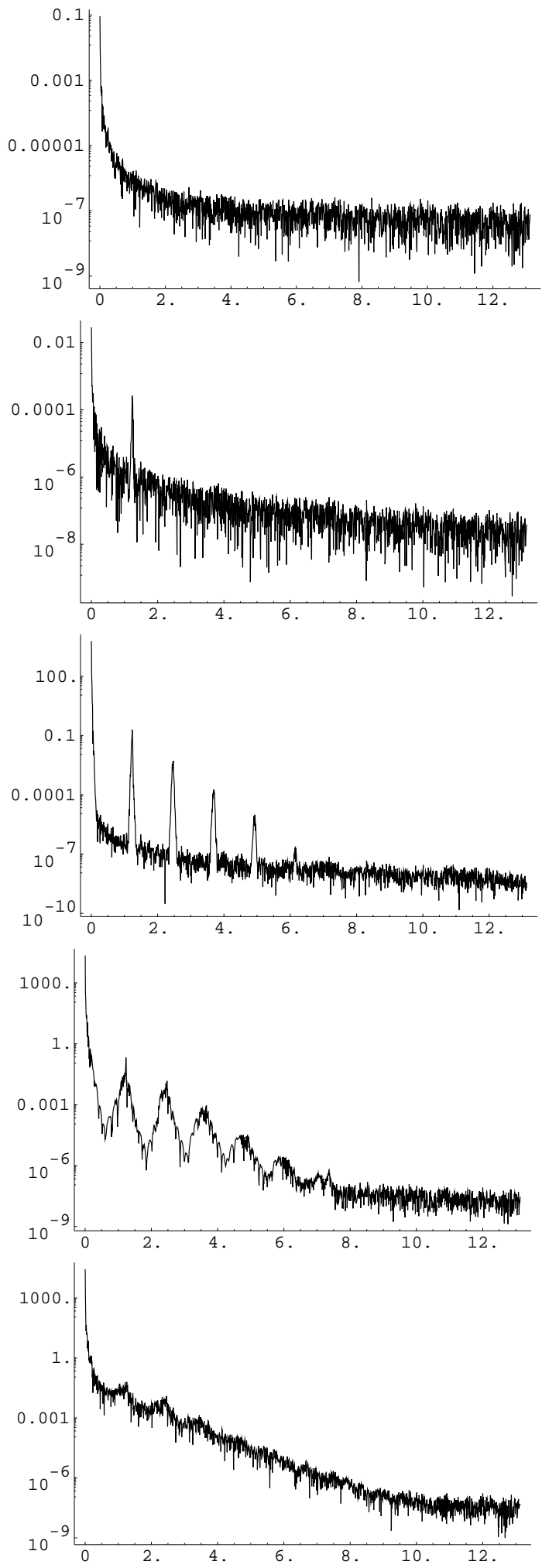

FIG. 4. The power spectrum, $\left|\Phi_{k}\right|$, of the gauge-invariant metric perturbation is plotted against $k$, with all modes initially excited. The spectra are plotted at $\eta=0.0183,274.5$, $439.2,750.3$ and 915 , respectively. 

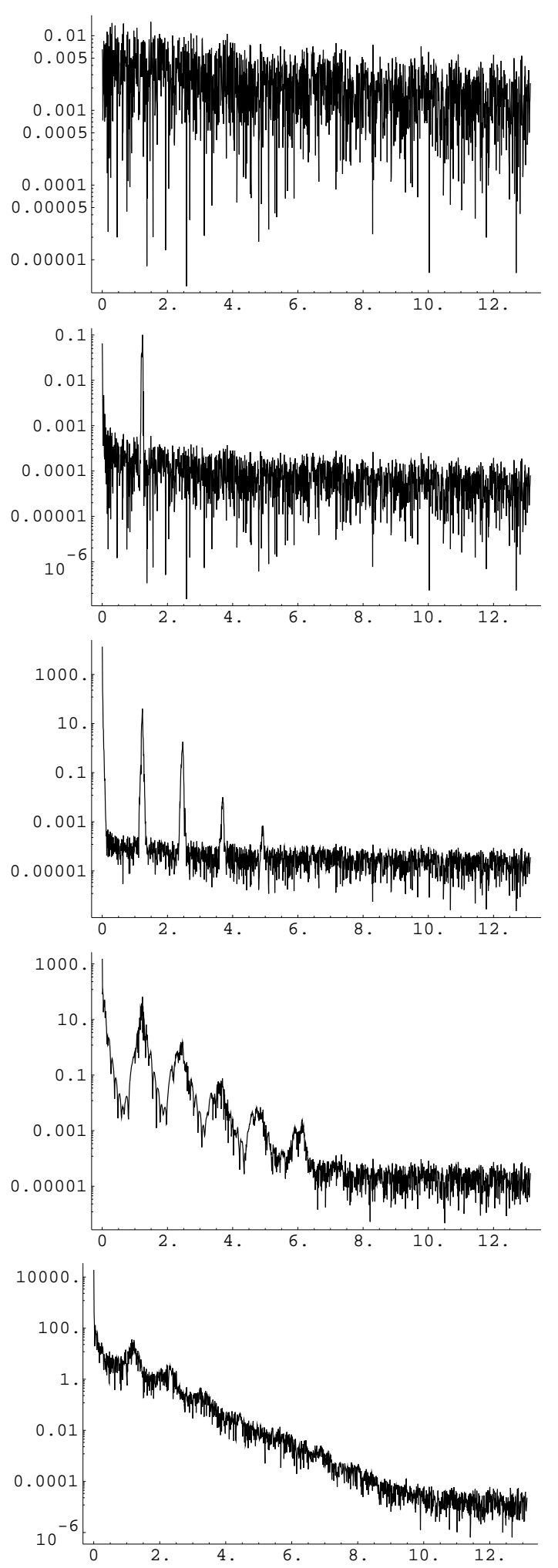

FIG. 5. The power spectrum of $\left|Q_{k}\right|$, is plotted against $k$, with all modes initially excited. The spectra are plotted at the same times as in fig. (价).
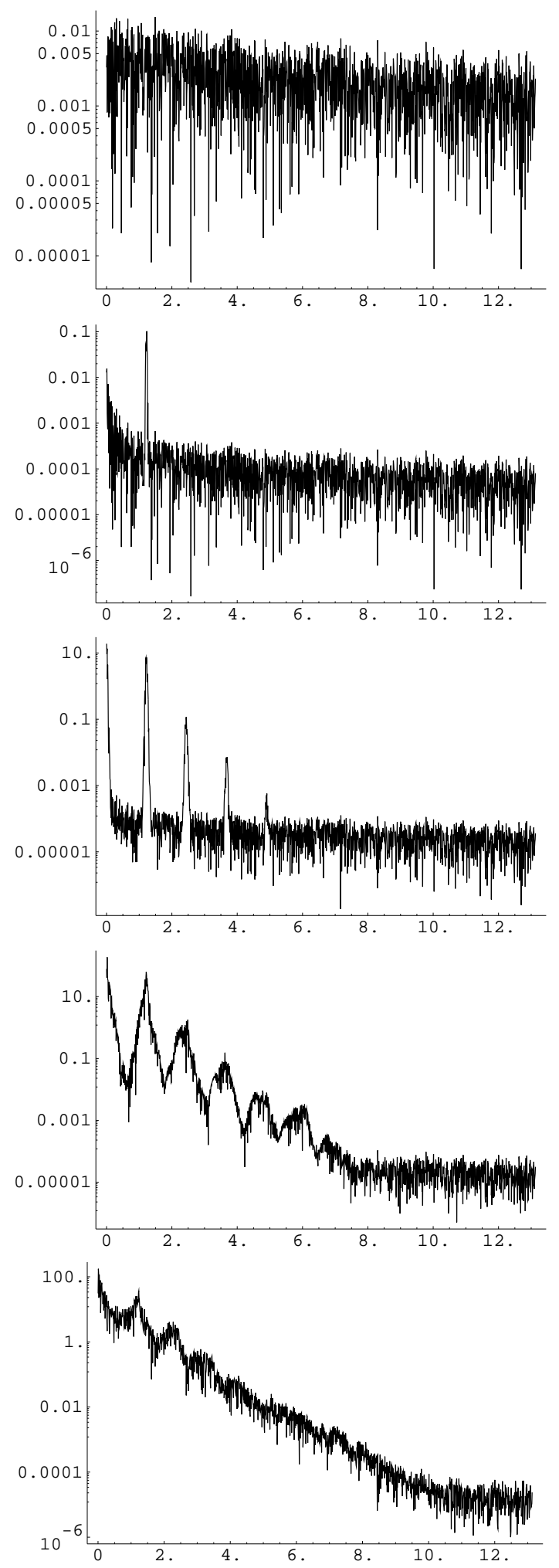

FIG. 6. The power spectrum of $\left|\chi_{k} / a\right|$, derived from a solution of equation (12), is plotted against $k$, with all modes initially excited. The spectra are plotted at the same times as in fig. (4) and fig. (5). 


$$
\begin{gathered}
N=262144=2^{18}, \delta \eta=\delta \zeta=0.0183 . \\
\phi_{0}=-0.58132, \dot{\phi}_{0}=0
\end{gathered}
$$

The actual volume is the same as in the previous simulation, since the higher value of $N$ is balanced by the lower value of $\delta \zeta$, but the spatial resolution is improved.

The amplitude of the initial perturbation here is significantly larger than the value obtained by extrapolating the COBE normalization to the scales probed by our simulation. We made this choice to reduce the time required for nonlinear effects to become important. However, apart from prolonging the epoch during which the perturbative approximation is valid, repeating the calculation with an initial inhomogeneity that is 100 times smaller does not appear to make any significant difference to our results.

The most important difference between the multimode case and the previous computation is in the behavior of the longest wavelength modes. Recall that in the linear approximation (and in the absence of a transient, decaying solution), and with $k \ll a H$,

$$
Q \propto \frac{\dot{\phi}}{H}, \quad \Phi \propto 1-\frac{H}{a} \int_{t_{0}}^{t} a\left(t^{\prime}\right) d t^{\prime},
$$

so that $\mathrm{Q}$ is oscillatory. With a single excited mode nonlinear couplings amplified higher harmonics of the resonant mode, but the the long wavelength (low $k$ ) modes did not grow, and obey the relationships in equation (51). Conversely, with all the modes initially excited the longest modes undergo considerable growth, implying that the linearity assumption of equation (51) is violated, and that mode-mode effects make a large contribution to the evolution of the longest modes. In fig. (4) we plot $\left|\Phi_{k}\right|$ for the multi-mode case at five different times during the simulation. Initially, only resonant modes grow significantly. However, once nonlinear effects begin to transfer power to the harmonics of the modes in the resonance band, we see that modes near $k=0$ also start to grow. The resonant growth terminates, but the "bands" continue to broaden, and eventually merge to produce a continuous spectrum. Finally, at the end of resonance the $\chi_{k} / a$ modes do not decay, as they did when only a single mode was excited.

We understand the evolution as follows. The initial growth of modes in the resonance band is explained by the perturbative analysis. The subsequent enhancement of the harmonics and the modes near $k=0$ is due to mode-mode couplings. Second order terms transfer power from modes labeled by $k_{1}$ and $k_{2}$ to the modes with $k_{1}+k_{2}$ and $\left|k_{1}-k_{2}\right|$. With just one excited mode, $k_{1}=k_{2}$, so we can produce the mode-doubling seeing in the previous simulation. However, for a single mode $k_{1}-k_{2}=0$, and modes with low $k$ are not excited. Finally, the broadening of the "bands" is (at minimum) a third-order effect. For instance, given three modes in one of the narrow bands, combinations like $k=k_{1}+k_{2}-k_{3}$, transfer power to modes adjacent to the band. This accounts for the observation that the higher harmonics are excited well before the bands themselves start to broaden.

We also note that there is an alternative hypothesis which might explain the enhancement of the long wavelength modes. A conventional perturbative calculation predicts that these modes do not undergo resonance, but this calculation ignores the back reaction of the resonant perturbations on the oscillating homogeneous mode. This will induce a subtle change in the forcing term in the perturbation equations, which is provided by the oscillating field, and this correction could conceivably induce new resonances at long wavelengths. This is consistent with our results, since a single excited mode will have a much smaller back reaction than that produced by the full spectrum of excited modes, accounting for the absence of this effect in the single mode simulation. Moreover, the resonance structure of $\lambda \phi^{4}$ inflation is known to be very sensitive to small changes in the driving function. If this is correct, the harmonics of the resonant modes would still be excited through mode-mode couplings, but the longest modes would be excited by a co-operative effect which would involve all the resonant modes. We stress that we have not yet attempted to verify this hypothesis. Note too that the possibility of backreaction by perturbations on the homogeneous mode is discussed (albeit in different models) in [27.28].

The most important question is the extent to which these effects are attributable to nonlinear gravitational couplings, as opposed to the nonlinear couplings provided by the $\lambda \phi^{4}$ potential. Looking at the solutions of $\chi / a$ found via equation (12), we see a similar, but not identical, resonance structure to that predicted by relativistic calculation. The discrepancy is most obvious at long wavelengths. In fig. (5) we plot $Q_{k}$ and in fig. (6) we show $\chi_{k} / a$ at the same times as the plots in fig. (14).

It is more appropriate to compare $\chi / a$ to $Q$ (or $\delta \phi^{(\mathrm{gi})}$ ) than to $\Phi$, since the latter vanishes if the metric is unperturbed. At short wavelengths, the $Q_{k}$ and $\chi_{k} / a$ are very similar, but at the longest wavelengths $Q_{k}$ and $\delta \phi^{\text {(gi) }}$ undergo much more growth than $\chi_{k} / a$. Moreover, while the longest modes of $\chi_{k} / a$ do grow, albeit less dramatically than the corresponding $Q_{k}$, the growth begins significantly later. This is illustrated by fig. (7), which plots the evolution of the longest modes as a function of $\eta$. Conversely, fig. (8) shows the same plot for $k=50$, and in this case the difference between the evolution of $\chi / a$ and the relativistic quantities is much less pronounced. The final plot in fig. (7) shows the growth of $Q$ for a mode inside the resonance band (this is actually the mode that was excited in the single mode case). Looking at this plot, we see that the near exponential growth of the long-wavelength modes ceases when the resonance is terminated.

We complete our analysis of figs (7) and (8) by numerically estimating the slopes of the the exponential segments of the plots. These slopes contain an arbitrary multiplicative factor, fixed by the normalization of $a$, 
which enters via the definition of the conformal time, $\eta$. Numerically, and with our normalization of $\eta$, we find that the exponential segments of the first four plots in fig. (7) have a slope of approximately 0.036. Conversely, the resonant mode shown in the bottom panel has a slope that is half of this value (0.017), supporting our contention that the growth in the longest modes is due to nonlinear couplings between two adjacent resonant modes. Finally, the exponential segment of the plots in fig. (8) has a slope of around 0.08 , confirming that this growth is driven by a strongly nonlinear multi-mode coupling.

Since we are working with the full Einstein field equations we can examine non-perturbative measures of curvature. For instance, $C^{2}=C_{\mu \nu \gamma \lambda} C^{\mu \nu \gamma \lambda}$, where $C$ is the usual Weyl tensor, is a covariant scalar density that is sensitive to the local spacetime curvature. Consequently, we can define

$$
\begin{aligned}
\mathcal{C}^{2}(\eta) & =\int d \zeta \sqrt{-g} C^{2}(\zeta, \eta) \\
\mathcal{R}^{2}(\eta) & =\int d \zeta \sqrt{-g} R^{2}(\zeta, \eta)
\end{aligned}
$$

where $R$ is the usual curvature scalar. These integrals are not covariant, since they depend on a specific co-ordinate choice. However, $\mathcal{C}^{2} / \mathcal{R}^{2}$ is dimensionless, and gives a qualitative measure of the inhomogeneity on our hypersurfaces, which we plot in fig. (10), because $C^{2}$ vanishes everywhere in an FRW spacetime. Thanks to our choice of initial conditions, the metric functions are constants at $\eta=0$, so $C^{2}$ vanishes on the initial hypersurface. Hence, the magnitude of $C^{2} / R^{2}$ is a measure of how far our spacetime departs from a perfectly FRW universe. The initial entropy perturbation quickly generates a metric perturbation. This remains constant until the nonlinear growth of the longest modes sets in at around $\eta=200$, whereupon $\mathcal{C}^{2} / \mathcal{R}^{2}$ grows exponentially until the underlying resonance is halted. However, after resonance ends $\mathcal{C}^{2} / \mathcal{R}^{2}$ remains roughly constant.

If the enhanced density perturbations generated during resonance led to localized gravitational collapse and the formation of primordial black holes (or "black walls" in the case we consider here), this would be obvious from $\mathcal{C}^{2} / \mathcal{R}^{2}$ as the curvature would diverge in a collapsing region. Since this does not happen in our simulation we find no evidence that preheating after $\lambda \phi^{4}$ inflation leads to primordial black hole formation. However, this result should be interpreted with care. After inflation, the equation of state resembles a relativistic gas, where $p=\rho / 3$, and as a consequence gravitational collapse is much less efficient than in dust filled universe with $p=0$ 团 Con-

\footnotetext{
${ }^{\ddagger}$ Recall the absence of structure formation in a hot dark matter dominated universe, as compared to a cold dark matter universe with the same primordial perturbation spectrum.
}
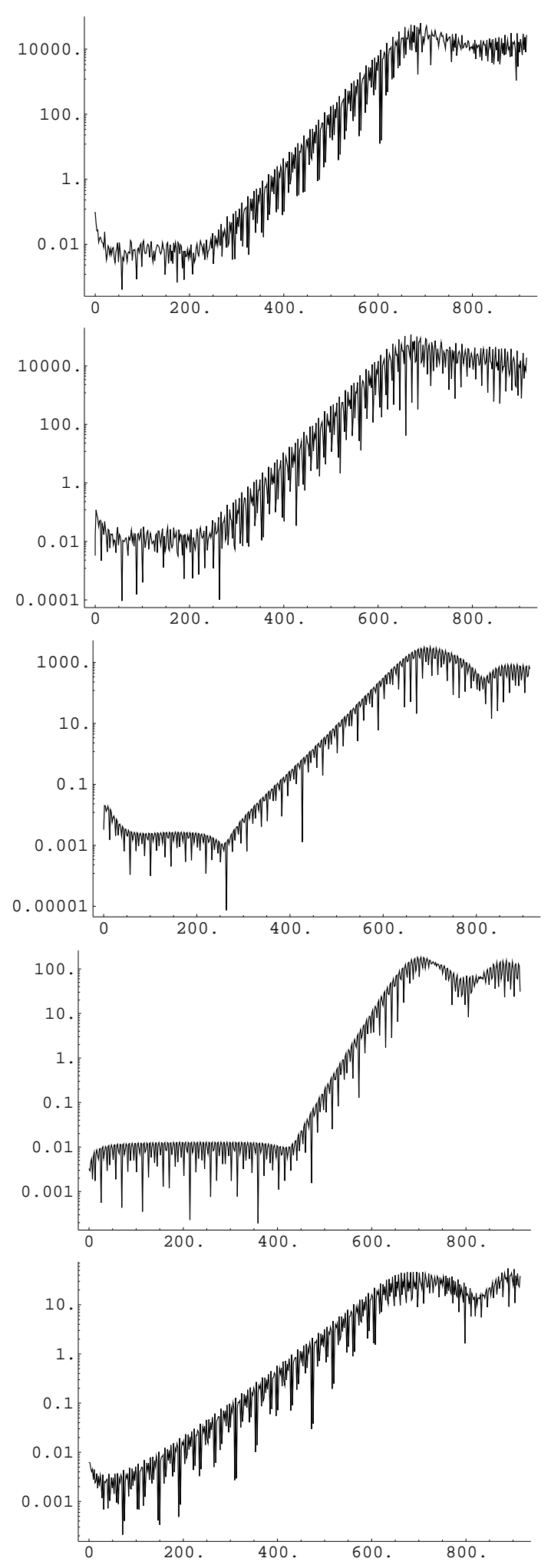

FIG. 7. From top to bottom, these plots show $\left|\Phi_{1}\right|,\left|Q_{1}\right|$, $\left|\delta \phi_{1}\right|,\left|\delta \chi_{1} / a\right|$ and $\left|Q_{583}\right|$ as functions of $\eta$. 

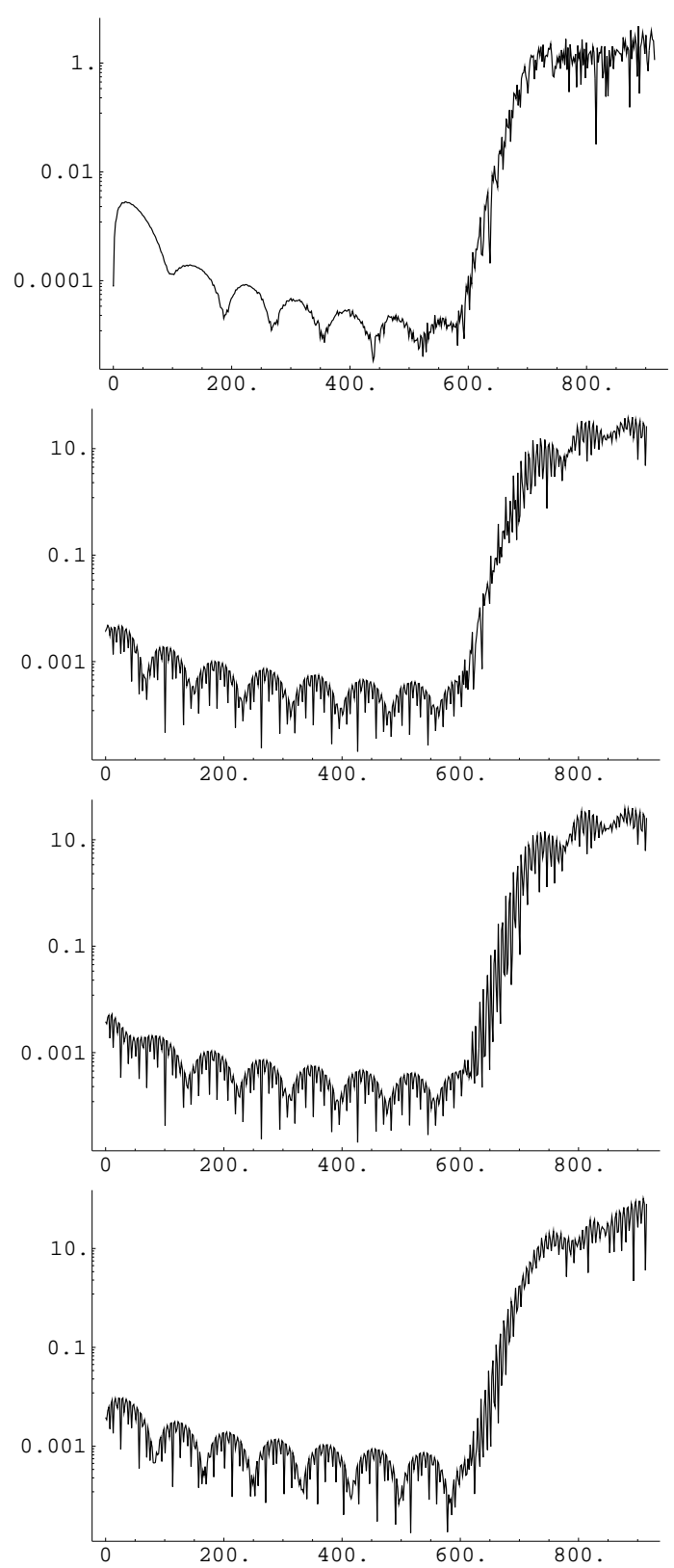

FIG. 8. From top to bottom, these plots show $\left|\Phi_{50}\right|,\left|Q_{50}\right|$, $\left|\delta \phi_{50}\right|$, and $\left|\delta \chi_{50} / a\right|$ as function of $\eta$.

sequently, even if preheating does sometimes lead to primordial black holes, it would not be surprising that they were absent from the $\lambda \phi^{4}$ case. In future work we will study more general potentials, and will be able to consider this topic in greater detail.

Apart from the length scale provided by the resonance band, the other important scale in the post-inflationary universe is the Hubble scale, $H^{-1}$. However, this length is not constant during the course of the simulation. In fig. (9) we plot the scale factor corresponding to the unperturbed FRW limit of our simulation, and we see that

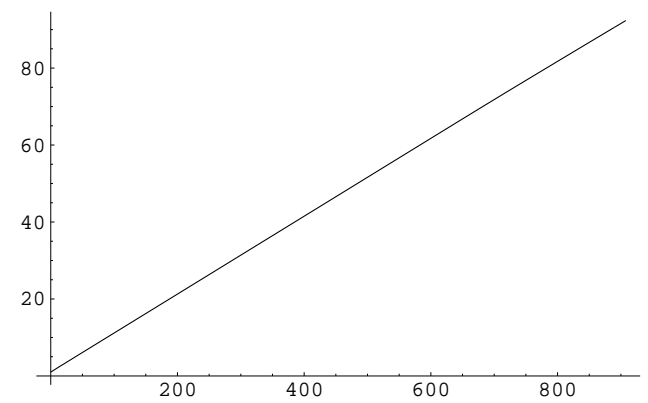

FIG. 9. This plot shows the scale factor $a(\eta)$, obtained by averaging the metric functions obtained from our simulation to remove the perturbation.

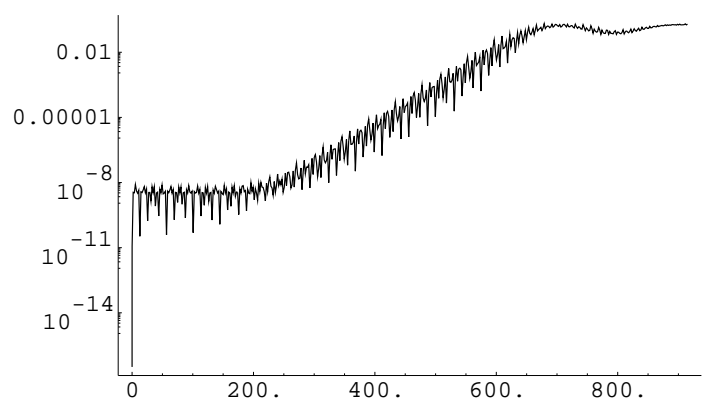

FIG. 10. The growth of $\mathcal{C}^{2} / \mathcal{R}^{2}$ during the course of the simulation is plotted as a function of $\eta$.

the universe grows 90 times larger during the simulation. During the same interval the Hubble radius grows linearly from an initial value of 10 to a final value of 900 (in our units). Our simulation region has a comoving width of $4797\left(2^{18} \delta \zeta\right)$, so the $k$-th mode has a comoving wavelength $4797 / k$. Modes with $k \lesssim 500$ are initially outside the horizon. At the time when the $k=1(\eta \sim 200)$ mode begins to grow, only modes with $k \lesssim 25$ are outside the Hubble horizon. By the end of the simulation, only four or five modes have wavelengths greater than $H^{-1}$.

Modes with $k \gtrsim 50$ show few differences between the evolution of $Q_{k}$ obtained from the relativistic calculation, and the analogous result for $\chi_{k} / a$. Consequently, on these scales (which can safely be termed "sub-Hubble") we see no evidence that nonlinear gravitational effects influence the overall progress of preheating after $\lambda \phi^{4}$ inflation.

For the longest modes there are clear differences between the results found in a fixed FRW background and the full relativistic calculation. Both the relativistic calculation and the nonlinear field equations in an unperturbed background predict that the longest modes of the field perturbation grow significantly. However, the relativistic results predict that this growth begins earlier and is an order of magnitude larger than that found from the field evolution in a rigid background. Note that in both 


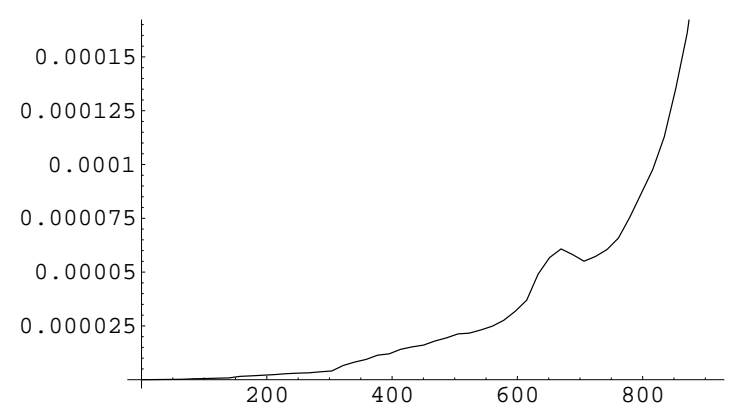

FIG. 11. The evolution of $\gamma$, equation (54), which quantifies the accuracy with which we satisfy the constraint, equation (27), is plotted for our numerical solution. For an exact solution $\gamma$ would be identically zero.

cases we are seeing the growth of modes that lie outside the instantaneous Hubble horizon.

At present, we do not identify the source of this discrepancy, but several possibilities exist. The most interesting explanation would obviously be back reaction, or some other nonlinear effect [22,25,29,30]. However, we stress that we have no evidence that the specific results of our simulations are predicted by these authors.

There are a number of less glamorous possible explanations for the enhanced inhomogeneity, which we briefly review. Firstly, it could be a side effect of using periodic boundary conditions, which induce an artificial longrange correlation into our simulation. Similarly, we assume that the inhomogeneity is limited to one spatial dimension, and the nonlinear effects we observe may be an artifact of this restriction. In many nonlinear systems the dimensionality has a crucial influence on the behavior, so we would still need to verify that any cosmologically significant changes to long wavelength modes persisted in the fully inhomogeneous case. On the other hand, our calculation of $\chi / a$ is also restricted to a single inhomogeneous dimension, so the dimensional dependence of $Q$ and $\chi / a$ would have to be different if this explanation is correct. Likewise, the three dimensional simulations of Khlebnikov and Tkachev [7], show a band structure that is qualitatively similar to that obtained here for $\chi / a$, which suggest that at shorter wavelengths our results are reliable. However, since we consider with a $1+1$ dimensional system we are able to resolve a large range of length-scales, whereas the simulation region in Khlebnikov and Tkachev's work is not significantly larger than the initial Hubble volume. Consequently, we cannot compare our calculation to theirs at super-Hubble scales.

We also need to be sure that the results we have seen do not depend on our introduction of the $\psi$ field to satisfy the initial constraint. Ideally in future work we will solve the constraints directly on the initial surface and dispense with $\psi$ entirely. However, we have varied the initial fraction of $\psi$ and checked that changing it made no significant difference to our results.
A different possibility is that our "reference" hypersurfaces, defined as the 3-planes with constant $\eta$, are degenerate in some way. If this is true the degeneracy must be shared by $t(\eta, \zeta)$ and $z(\eta, \zeta)$, which we compute from equations (41) and (42), as $z$ and $t$ remain smooth functions of $\zeta$ and $\eta$ during the simulation. Moreover, we have been careful to work with variables which are invariant (to first order) under gauge transformations.

Numerically, we checked our solutions by ensuring that they did not change significantly when we altered the parameters that govern the numerical integration scheme. In particular, the results appear to be independent of $N$, the number of points on our grid, the distance between them, $\delta \zeta$, and the timestep $\delta \eta$. The accuracy with which we fit the constraints provides an independent test of our results, and the constraints are indeed well satisfied, especially during the era when nonlinear effects first become apparent.

The constraints, equations (26) and (27), allow us to form an independent measure of the accuracy of our code. To measure how well our solution obeys a given constraint we evaluate

$$
\gamma^{2}=\frac{\int d \zeta(l-r)^{2}}{\sqrt{\int d \zeta l^{2}} \sqrt{\int d \zeta r^{2}}} .
$$

We have two different $\gamma$, corresponding to the two constraints, while $l$ and $r$ are the left and right hand sides of the equation equation (26) or equation (27) (depending on which constraint we are testing), and $\gamma$ is the average fractional difference between the left and right sides of the constraint equations. In fig. (11) we plot the $\gamma$ derived from equation (27), and we see that it is always small. Moreover, $\gamma$ decreases with $\delta \eta$ and $\delta \zeta$. In general, the value of $\gamma$ derived from equation (26) is smaller than that obtained from equation (27), and we do not show it here.

Our choice of $N$ and $\delta \zeta$ must satisfy two opposing criteria. Ideally, we would like to be able to resolve modes which were much larger than the Hubble radius for the entire duration of resonance, but to prevent the build up of numerical error we must resolve all the harmonics of the resonant modes that are excited by the nonlinear couplings. This sets an upper limit on $\delta \zeta$ and $\delta \eta$. Including super-Hubble modes sets a lower limit on the total size of the box. The combination of these two effects is to ensure that $N$ must be quite large. Consequently, while it is feasible to include modes which are a few times larger than the Hubble radius at the end of resonance, looking at modes 100 times larger (or more) would require a significant amount of computer time. A better approach may be to look for similar effects in models where the resonance is stronger, which means that the overall growth of the universe during the resonant era will be much smaller than in the $\lambda \phi^{4}$ case. 


\section{CONCLUSIONS}

We have analyzed parametric resonance after $\lambda \phi^{4}$ inflation for two distinct sets of initial conditions. In the first we gave only one Fourier mode of the perturbation a nonzero initial amplitude, and in the second all modes were initially excited. The overall picture of the resonant era derived from our calculations, which incorporates nonlinear gravitational effects, is similar to that obtained from analyses of the nonlinear field dynamics which assume that the background spacetime is rigid. However, in both simulations we saw new phenomena which appear to be due to nonlinear gravitational effects. With a single mode excited, the perturbation calculated in a rigid spacetime background decayed after the resonant growth terminated, but its amplitude after resonance remained large when nonlinear gravitational effects were included. When all modes were initially excited power was transferred to long wavelength modes by second order effects in both the rigid background and nonlinear gravitational calculations, but in the latter case the overall growth is larger and begins sooner. However, we do not see any evidence for the formation of primordial black holes and the long wavelength modes cease growing once the exponential increase of the modes inside the resonance band comes to a halt.

Given the recent interest in nonlinear gravitational effects during preheating and in gravitational back reaction, the enhanced inhomogeneity we see at long wavelengths when all modes are initially excited is a tantalizing result. However, more work will be needed to confirm that these effects have a physical origin, and are not an artifact of our numerical scheme or underlying assumptions. Moreover, if the effect is real, its precise origin and physical consequences remain to be determined. In addition, the symmetry condition we have imposed in our simulation can be shown to preclude the existence of tensor perturbations to the metric, so we cannot reach any conclusions about the resonant production of gravitational waves after inflation.

Finally, the numerical codes and analytic techniques developed during this investigation are readily applicable to other resonant models, including those based on two (or more) interacting scalar fields. Since the structure of the resonance after $\lambda \phi^{4}$ inflation is very simple, we can form a clear picture of the evolution of the inhomogeneous parts of the field and the spacetime metric during the resonant era. Consequently, in addition to its intrinsic interest, $\lambda \phi^{4}$ is an excellent model to begin with as it has allowed us to perfect the techniques we will use in the analysis of other, more complicated, systems. This work is currently in progress, and its results will shed further light on the potential nonlinear gravitational effects we have uncovered here.

\section{ACKNOWLEDGMENTS}

We thank Robert Brandenberger, Fabio Finelli and Andrew Sornborger for useful discussions. Computational work in support of this research was performed at the Theoretical Physics Computing Facility at Brown University. RE and MP were supported by DOE contract DE-FG0291ER40688, Task A.

[1] J. H. Traschen and R. H. Brandenberger, Phys. Rev. D 42, 2491 (1990).

[2] D. Polarski and A. A. Starobinsky, Nucl. Phys. B 385, 623 (1992).

[3] L. A. Kofman, A. D. Linde, and A. A. Starobinsky, Phys. Rev. Lett. 73, 3195 (1994).

[4] Y. Shtanov, J. Traschen, and R. Brandenberger, Phys. Rev. D 51, 5438 (1995).

[5] T. Prokopec and T. G. Roos, Phys. Rev. D 55, 3768 (1997).

[6] D. Kaiser, Phys. Rev. D 53, 1776 (1996).

[7] S. Y. Khlebnikov and I. I. Tkachev, Phys. Rev. Lett. 77, 219 (1996).

[8] S. Y. Khlebnikov and I. I. Tkachev, Phys. Rev. Lett. 79, 1607 (1996).

[9] S. Y. Khlebnikov and I. I. Tkachev, Phys. Lett. B 390, 80 (1996).

[10] L. Kofman, A. Linde, and A. Starobinsky, Phys. Rev. D 56, 3258 (1997).

[11] P. B. Greene, L. Kofman, A. Linde, and A. A. Starobinsky, Phys. Rev. D 56, 6175 (1997).

[12] B. R. Greene, T. Prokopec, and T. Roos, Phys. Rev. D 56, 6484 (1997).

[13] H. Kodama and T. Hamazaki, Prog. Theor. Phys. 96, 949 (1996).

[14] T. Hamazaki and H. Kodama, Prog. Theor. Phys. 96, 1123 (1996).

[15] E. W. Kolb, A. D. Linde, and A. Riotto, Phys. Rev. Lett. 77, 4290 (1996).

[16] D. Kaiser, Phys. Rev. D 56, 706 (1997).

[17] D. Kaiser, Phys. Rev. D 57, 702 (1997).

[18] Y. Nambu and A. Taruya, Prog. Theor. Phys 97, 83 (1996).

[19] Y. Nambu and A. Taruya, Class. Quantum. Grav. 15, 2761 (1998).

[20] A. Taruya and Y. Nambu, Phys. Lett. B 428, 37 (1998).

[21] E. W. Kolb, A. Riotto, and I. Tkachev, Phys. Lett. B 423, 348 (1998).

[22] B. Bassett, D. Kaiser, and R. Maartens, Phys. Lett. B 455, 84 (1999).

[23] F. Finelli and R. Brandenberger, Phys. Rev. Lett. 82, 1362 (1999).

[24] M. Parry and R. Easther, Phys. Rev. D 59, 061301 (1999). 
[25] B. Bassett, F. Tamburini, D. Kaiser, and R. Maartens, hep-ph/9901319 (1999).

[26] K. Jedamzik and G. Sigl, hep-ph/9906287 (1999).

[27] B. A. Bassett and F. Viniegra, hep-ph/9909353 (1999).

[28] B. A. Bassett, C. Gordon, R. Maartens and D. I. Kaiser, hep-ph/9909482 (1999).

[29] V. Mukhanov, L. R. W. Abramo, and R. Brandenberger, Phys. Rev. Lett. 78, 1624 (1997).

[30] L. R. W. Abramo, R. H. Brandenberger, and V. F. Mukhanov, Phys. Rev. D 56, 3248 (1997).

[31] V. Mukhanov, H. Feldman, and R. H. Brandenberger, Phys. Rep. 215, 203 (1992).

[32] V. F. Mukhanov, Sov. Phys. JETP 68, 1297 (1988).

[33] J. Hwang, gr-qc/9608018 (1996).

[34] J. García-Bellido, A. Linde, and D. Wands, Phys. Rev. D 54, 6040 (1996).

[35] B. J. Carr, J. H. Gilbert, and J. E. Lidsey, Phys. Rev. D 50, 4853 (1994).

[36] A. Green and A. Liddle, Phys. Rev. D 56, 6166 (1997).

[37] R. Easther and M. Parry, hep-ph/9902088 (1999).

[38] M. Parry and R. Easther, hep-ph/9903550 (1999).

[39] J. York, in Gravitational Radiation, Proceedings of Les Houches, edited by N. Deruelle and T. Piran (NorthHolland Publishing, Amsterdam, 1983).

[40] D. S. Goldwirth and T. Piran, Phys. Rep. 214, 223 (1992).

[41] M. W. Choptuik, Phys. Rev. D 44, 3124 (1991).

[42] D. Gottlieb and S. Orszag, Numerical analysis of spectral methods: theory and applications (SIAM, Philadelphia, 1977). 